\title{
Expanding the Understanding of Content of End-of-Life Dreams and Visions: A Consensual Qualitative Research Analysis
}

\author{
Rachel M. Depner, MS, ${ }^{1,2}$ Pei C. Grant, PhD, ${ }^{1, *}$ David J. Byrwa, MS, ${ }^{1,3}$ Sarah M. LaFever, PhD, 4 \\ Christopher W. Kerr, MD, PhD, ${ }^{1}$ Kelly E. Tenzek, PhD, ${ }^{5}$ Susan LaValley, PhD, ${ }^{6}$ Debra L. Luczkiewicz, MD, ${ }^{1}$ \\ Scott T. Wright, PhD, ${ }^{7}$ and Kathryn Levy, MSW AdvStat ${ }^{1,8}$
}

\begin{abstract}
Background: Research has established End-of-Life Dreams and Visions (ELDVs) as prevalent, meaningful valid experiences that may help patients cope with illness and approaching death. However, no inductive qualitative analysis has explored the phenomenology of ELDVs from the perspective of hospice homecare patients.

Objective: The purpose of this study is to evaluate the content of ELDVs by using a rigorous qualitative approach. Design: Five hundred forty-eight ELDVs were collected from weekly interviews of hospice homecare patients and analyzed by using Consensual Qualitative Research Methodology.

Settings/Subject: Participants were enrolled in a county-wide hospice homecare program between January 2013-March 2015.

Results: The following domains emerged: (1) Interpersonal, (2) Affective Experience and Reflection, (3) Activities, and (4) Setting/Location.

Conclusions: This study suggests that ELDV content may include a broader spectrum of experiences that reflect waking life than previously believed. Clinical implications suggest that it may be important for providers to engage with ELDVs, as they are psychologically significant experiences that may be a source of clinical insight.
\end{abstract}

Keywords: consensual qualitative research; end-of-life dreams and visions; hospice care

\section{Introduction}

Research has shown that subjective experiences such as end-of-life dreams and visions (ELDVs) play an important role in the dying trajectory by offering comfort, psychological/spiritual solace, and meaning-making. ${ }^{1-15}$ The ELDVs involve mental and sensory activity while the patient is asleep (dreams) or awake (visions) and are typically reported to include seeing or feeling the presence of deceased loved ones. Research has evolved from case-based anecdotes and caregiver secondhand accounts ${ }^{4,5,9,11,13-17}$ to reports of patient perspec- tives. ${ }^{1,2,10}$ More recently, the scope of ELDV research has expanded to explore the impact on post-traumatic growth of patients ${ }^{6}$ and bereavement outcomes of familial caregivers. ${ }^{18}$

The ELDVs have often been dismissed by medical professionals as alterations in cognition (e.g., delirium). Although the dying process is frequently complicated by delirium, ${ }^{19-21}$ there are key elements that distinguish ELDVs from this type of cognition (Table 1). Delirium includes a breakdown in attention and awareness, ${ }^{22}$ whereas individuals experiencing

\footnotetext{
${ }^{1}$ Department of Research, Hospice and Palliative Care Buffalo, Cheektowaga, New York, USA.

${ }^{2}$ Department of Counseling, School and Educational Psychology, ${ }^{3}$ Jacobs School of Medicine and Biomedical Sciences, ${ }^{5}$ Department of Communication, and ${ }^{6}$ Primary Care Research Institute, Department of Family Medicine, University at Buffalo, The State University of New York, Buffalo, New York, USA.

${ }^{4}$ Behavioral VA Health Care Line (BVAC), VA Western New York Healthcare System, Buffalo, New York, USA.

${ }^{7}$ University Counseling Center, University of Rochester, Rochester New York, USA.

${ }^{8}$ Department of Planning and Research, Trocaire College, Buffalo, New York, USA.

*Address correspondence to: Pei C. Grant, PhD, Department of Research, Hospice and Palliative Care Buffalo, 225 Como Park Boulevard, Cheektowaga, NY 14227, USA, E-mail: pgrant@palliativecare.org
}

(c) Rachel M. Depner et al., 2020; Published by Mary Ann Liebert, Inc. This Open Access article is distributed under the terms of the Creative Commons License (http://creativecommons.org/licenses/by/4.0), which permits unrestricted use, distribution, and reproduction in any medium, provided the original work is properly cited. 
Table 1. Conceptualization and Differentiation of End-of-Life Dreams and Visions and Delirium

\begin{tabular}{|c|c|c|}
\hline Sphere of being & Delirium & ELDV \\
\hline Physical & Agitated movements, picking & Mild at rest \\
\hline Cognitive & Disorganized, confused & $\begin{array}{l}\text { Heightened acuity } \\
\text { and recall }\end{array}$ \\
\hline Relational & Withdrawn, disconnected & Engaged, connected \\
\hline Emotional & Distressed & Comforted, calm \\
\hline Mental & Sense of disbelief & Sense of reality \\
\hline $\begin{array}{l}\text { Existential/ } \\
\text { spiritual }\end{array}$ & $\begin{array}{l}\text { Disconnected from } \\
\text { spiritualty }\end{array}$ & $\begin{array}{l}\text { Peaceful, } \\
\quad \text { transcendent }\end{array}$ \\
\hline
\end{tabular}

This table demonstrates the multifaceted clinical differences between the phenomenon of ELDVs and the experience of delirium; it is meant to be used as an educational but not diagnostic tool.

ELDVs, end-of-life dreams and visions.

ELDVs typically demonstrate organized thoughts. ${ }^{1,10}$ This distinction is clinically important, as medical professionals can play an important role facilitating meaning and comfort by not unnecessarily medicating patients with ELDVs. Professionals' failure to understand this distinction may even prevent the patient from engaging in a meaningful experience that is intrinsic to the dying process.

The ELDVs may serve as a psychological mechanism that helps patients process and/or cope with approaching death. ${ }^{7,23}$ Although typically meaningful and calming, ELDVs can be potentially distressful, ${ }^{2,24}$ potentially even more for those with a history of trauma, comorbid mental health disorders, and/or increased existential suffering. Dream experiences may mirror waking life experiences in healthy ${ }^{25,26}$ and chronically ill/dying individuals, ${ }^{7,23,25-27}$ and they can be used as a nonthreatening avenue for engaging in difficult conversations about death/dying or past experiences. Although one study has explored how the interpretation of ELDV content might facilitate meaning-making at the end of life, ${ }^{7}$ much remains unknown about the phenomenological experience of ELDVs. A more thorough understanding of ELDV content might inform further development of psycho-social-spiritual treatment for dying patients. Therefore, the goal of this study was to evaluate the content of ELDVs as reported by patients enrolled in hospice homecare by using consensual qualitative research (CQR) methodology.

\section{Methods}

This is a qualitative analysis of 548 ELDV reports from 55 people enrolled in a hospice homecare program. The study was approved by the Social and Behavioral Research Institutional Research Board of a midsize public university in New York (FWA00008824) on 11/14/2012.

\section{Participants}

Patients. Participants were enrolled in a county-wide hospice homecare program between January 2013 and March 2015. Researcher visits occurred in patient residences. All participants were (1) currently enrolled in a hospice homecare program, (2) older than the age of 18, (3) able to provide consent, and (4) had a Palliative Performance Scale (PPS) ${ }^{28}$ score of at least $30 \%$. Patients were not eligible for this study if they had diagnoses affecting cognition or if there was a language barrier.

\section{Measures}

Demographics. Participants reported age, gender, race, marital status, and religious affiliation. The PPS and additional relevant diagnostic data were collected from electronic medical records (EMRs).

Cognitive and health status. Clinical information was collected and reviewed before each study visit. The confusion assessment method (CAM), a validated clinical tool that assesses delirium, ${ }^{21,29}$ was administered to assess appropriateness for ongoing participation.

Semi-structured dream interview. A semi-structured interview was developed for this study based on previous research ${ }^{1,2}$ and clinical experience. Interviews were only administered after participants reported at least one ELDV (Table 2).

\section{Procedures}

Participants were recruited based on clinician referral or review of EMRs. Individuals meeting criteria were given the opportunity to participate regardless of

\section{Table 2. Structured Dream Interview Questions}

\section{Question}

Please tell me about your dream/vision.

Who or what are you seeing in these dreams or visions?

What were the people (or figures, animals) in your dreams (or visions) doing?

How did you feel about this dream or vision? Or what was your reaction to this dream or vision?

How do these dreams or visions impact your sense of meaning and purpose of your life?

How do these dreams or visions impact your understanding of your relationships?

Do these dreams or visions affect the way you see death and dying? If so, how?

Does this dream or vision affect any unresolved issues in your life?

Were you or the people (or figures, animals) in your visions (or dreams) going somewhere or preparing to go somewhere?

This table includes all structured questions utilized in the interview process but does not include unique follow-up probes. These questions are derived from previous research ${ }^{1,2}$ and clinical experience. 
whether they reported ELDVs or not. Verbal consent was reobtained at each visit. Participants were not provided any additional benefits or compensation. All participants were given a notebook to optionally record ELDVs. Weekly data collection was completed by a researcher or clinician from the time of consent until participants were unable to/chose not to continue. At the start of each weekly visit, the CAM was administered to ensure that the participant was not delirious. All data from semi-structured interviews were transcribed verbatim and de-identified.

\section{Data analysis}

The CQR methodology was used to analyze data. ${ }^{30,31}$ This analysis incorporates elements of both constructivism and post-positivism as the primary research paradigm by using an inductive, systematic qualitative analysis that adopts a team consensus approach. A core team and external auditors engage in all steps of the process. The process includes (1) sorting data into domains or overarching themes, (2) converting raw data into core ideas, and (3) cross-analysis, which evaluates within each domain for emergent categories (or subthemes). Representativeness across all categories is calculated. Representativeness was calculated per CQR guidelines by the number of participants who described at least one ELDV within that category. All/all but one participant= general representativeness, between half and all=typical, more than four but less than half=variant, and less than three participants $=$ rare.

In this study, four researchers (R.M.D., P.C.G., D.J.B., S.M.L.) comprised the core data analysis team. Personal biases were recorded, discussed, and reported in Supplementary Data. One auditor (K.E.T.) reviewed the work of the core team. At each step of the CQR process, team members individually reviewed the data, then reviewed as a team, and finally reached an agreement before moving on to the next step. At each stage, the external auditor reviewed and provided feedback, which is incorporated at the discretion of the core team.

To limit bias, data were randomized so that proximity to death relative to reported ELDV was unknown. On completion of analysis, data were reordered based on days before death and categories and subcategories were analyzed for frequency.

\section{Results}

Demographics

Eighty-three patients participated in 842 weekly visits, resulting in 548 ELDV reports from 55 participants (66\%). Most participants were aged 61 to 101 years
(89\%), white/European (97\%), female (70\%), Christian $(82 \%)$, and with a primary diagnosis of cancer (47\%). The modal number of days of participation in the study was 14, but it ranged from 5 to 206 (mean 145 days; SD 123) (Table 3).

\section{CQR results}

Four domains emerged from the dataset: Interpersonal, Affective Experience and Reflection, Activities, and Setting/Location.

Interpersonal domain. The interpersonal domain was operationalized as an ELDV description featuring people/animals, and information about interactions. Within this domain two primary categories emerged (1) Characters and (2) Relational Interactions (Table 4).

Characters emerged with general representativeness, and they were quite diverse with 11 subcategories. In general, participants identified themselves as present within the ELDV. Typically, participants reported ELDV experiences featuring Family. One participant shared, "I see my mother and she talks to me."

It was also typical for ELDVs to include people who were Unfamiliar/Unknown. One participant reported, "Other people were standing around us but I did not recognize anyone else." Likewise, participants typically reported Miscellaneous Characters, such as healthcare providers, "stereotypical New York guys," people from TV commercials, and others. For example, "I remember Mrs. Peloquin, first person to be a weekly client. I did her hair for a very long time."

The final seven subcategories describing characters all emerged with variant representativeness. Peer

Table 3. Participant Demographic Data

\begin{tabular}{llrr}
\hline Characteristic & \multicolumn{1}{c}{ Group } & N & $\%$ \\
\hline \multirow{3}{*}{ Age } & Total & 55 & 100 \\
& $18-60$ & 6 & 11 \\
Gender & $61-101$ & 49 & 89 \\
& Female & 39 & 70 \\
Race & Male & 16 & 30 \\
& White/European & 53 & 97 \\
Diagnosis & African American & 2 & 3 \\
& Cancer & 26 & 47 \\
& COPD & 17 & 31 \\
Religious affiliation & 7 & 13 \\
& CHF & 5 & 9 \\
& Other & 45 & 82 \\
& Christian & 7 & 13 \\
& Atheist/none affiliated & 2 & 4 \\
& Jewish & 1 & 1 \\
\hline
\end{tabular}

This table displays the participant's self-reported demographic information, including age, gender, race, diagnosis, and religious affiliation.

$\mathrm{CHF}$, congestive heart failure; COPD, chronic obstructive pulmonary disease. 


\section{Table 4. Interpersonal Domain Categories and Subcategories with Case Representation and Frequency}

\begin{tabular}{lc}
\hline Category/subcategory & Frequency \\
\hline 1. Characters & General \\
Self-present in dream/vision & General \\
Family & Typical \\
Parents/parental figures & Variant \\
Siblings & Variant \\
Spouse/partner & Variant \\
Other relatives/extended family & Variant \\
Domestic pets & Variant \\
Unfamiliar/unknown & Typical \\
Miscellaneous characters & Typical \\
Peer relationship & Variant \\
Crowd/group & Variant \\
High profile/authority figures & Variant \\
Atypical/incongruent character descriptions & Variant \\
Characters who are alive again & Variant \\
Baby/child & Variant \\
Wildlife/unknown animals & Variant \\
2. Relational interactions & General \\
Close connection & Typical \\
Neutral connection & Typical \\
Alone & Variant \\
Disconnected/unable to connect & Variant \\
Moving toward & Variant \\
Conflictual connection & Variant \\
Moving away & Variant \\
\hline
\end{tabular}

This table reports the categories and subcategories from the interpersonal domain as well as representativeness in the dataset. The frequency labels are determined by CQR (Hill) ${ }^{30}$ and are as follows: General, the category is represented in all or all but one case; typical, occurs between half and less than all; and Variant, equals less than half but more than three cases.

$\mathrm{CQR}$, consensual qualitative research.

Relationships included friends, classmates, lovers, and co-workers and were distinguished by equivalent levels of power and social status. One participant shared, "I had a dream about a couple I used to be good friends with when my husband was alive." This contrasts with High Profile/Authority Figures, including characters beyond/above the power level of the dreamer who would not usually be in the same social circle. Atypical/Incongruent Character Descriptions included people who participants reported knowing, but whose characteristics did not match how they knew them in waking life, such as loved ones who appeared to have different physical characteristics, ages, or health status. One participant described, "I hear my three children playing up above me like when they were kids. I almost yell out and tell them to get to bed but then I realize they are all grown up." Similarly, participants reported experiencing ELDVs with Characters Who are Alive Again, including people who the participants know are already deceased. One said, "[I] dreamed about Mom and Dad; they were old in the dream but [are] deceased in life." Comparatively, participants were equally likely to discuss the presence of a baby/child, Groups/Crowds, and Wildlife/Unknown Animals.

Another category that emerged with general representativeness was Relational Interactions, which included information about exchanges during the ELDV between the participant and others. It was typical for participants to report interactions as Close Connections, or any relational description that is intimate and/or emotionally close. This encompassed feeling a presence that was positive, a sense of closeness, and/or connecting through verbal and nonverbal displays. One participant described feeling the warm presence of her husband, "[I am] very happy, especially at night, when I wake up and feel like he was snuggled up against me."

It was equally likely for participants to report ELDVs that included Neutral Connections. This interaction was differentiated as a lower level of relational engagement or not having a highly reactive tone, neither warm/intimate nor conflictual. For example, "My dad came to me in a dream and we were doing day-today things."

Interestingly, a few subcategories emerged that focused on the lack of interaction or a thwarted interaction. Examples include, "I was alone on a train." or "I am at a county fair with my parents and sister...I became annoyed with the family because they keep taking off for another area without letting me know." Another relational dynamic was variant Conflictual Connection, which includes relationships described as aggressive and/or hostile and ranged from minor conflict/ disagreement to full on physical/relational harm. One participant recalled, "Then he [dog] came up on the bed, looked right at me, smiled and peed. Then the last second of the dream was me chasing him through the house with a pot."

Affective experience and reflection. This domain emerged with a wide range of categories and subcategories, including two categories with general representativeness (Table 5).

Participants generally described their dreams with Feelings/Emotions. This is grounded primarily in direct statements such as "It made me feel happy" or "now that I think about it I am sad." Twelve main subcategories with variant representativeness emerged, including traditionally positive emotions such as: (1) peace/ calm/comfort, (2) nice/good/great, (3) happiness/ enjoyment/excitement/pleasure/fun, (4) humor/silly/ laughter; and (5) curiosity/wondering. One participant 
Table 5. Affective Experience Categories and Subcategories with Case Representation and Frequency

\begin{tabular}{lc}
\hline Category/subcategory & Frequency \\
\hline 1. Feelings/emotions & Typical \\
Peace, calm, comfort & Variant \\
Nice, good, great & Variant \\
Happiness, excitement, pleasure & Variant \\
Humor, silly, laughter & Variant \\
Curiosity, wondering & Variant \\
Neutral/no strong feelings, apathy & Variant \\
Disturbing, distressing, scary & Variant \\
Uncertainty, confused, puzzled & Variant \\
Anger, frustration, disappointment & Variant \\
Anxiety, stress, concern & Variant \\
Surprised, startled, shocked & Variant \\
Complex feelings & Variant \\
Sadness, sorrow, down & Variant \\
2. Reflections & Typical \\
Nostalgia & Typical \\
Meaning and Coherence & Typical \\
Dream Commentary & Typical \\
Felt odd, strange, weird & Variant \\
Real, vivid & Variant \\
Normal & Variant \\
\hline
\end{tabular}

This table reports the categories and subcategories from the Affective Experience domain as well as representativeness in the dataset. The frequency labels are determined by $\mathrm{CQR}$ (Hill) ${ }^{30}$ and are as follows: General, the category is represented in all or all but one case; typical, occurs between half and less than all; and Variant, equals less than half but more than three cases.

exemplified this, saying "it was comforting to see the trees and it was a beautiful fall day, I felt happy."

Equally, this category included emotions typically viewed as distressing: (1) uncertain/confused/ puzzled, (2) disturbing/distressing/scary/fearful/upset, (3) $\mathrm{mad} /$ angry/frustrated/disappointed/irritated, (4) worried/anxious/stressed/overwhelmed/concerned, (5) surprised/startled/shocked, and (6) sadness/sorrow/ blue. One participant reported a specific affective experience, "I was watching children play in my house and someone got hurt I was very upset by this. I woke up crying."

Two final subcategories within Feelings/Emotions were Neutral/No Strong Feelings and Complex Emotions. The latter was defined as the presence of multiple feelings within one dream that may be at odds with or contrast one another, adding complexity to the ELDV. Examples included, "I was surprised how accurate it [the dream] was. It was so good but it was also deeply, deeply disturbing."

The second category in the Affective domain is Reflection. Participants typically reflected on an element of Nostalgia, a longing to reconnect with a person, place, or experience from the past. One participant shared an ELDV of her friend who was deceased, "She is not interacting with me but I like having her there. When I wake up, I think, oh man, get back here!"

It was typical that participants reported at least one ELDV represented in the Meaning and Coherence category, in which they spontaneously engaged in sensemaking. This category is exemplified by the following report, “...my dreams have increased and I think I'm working things out in my dreams or trying to come to terms with my sickness in my dream." In addition, it was also typical that participants reported details or Dream Commentary about their ELDV experience. The remainder of subcategories in this section occurred with variant frequency.

Activities. This domain evaluates actions/events that occurred within the ELDV and resulted in 14 subcategories (Table 6). Typically, participants reported activities related to Traveling and Movement. One said, "I am on a bicycle coming down a steep curving mountain highway. I find that the speed of the bike is getting dangerously fast."

Similarly, participants typically reported Verbal Communication and Observing and Watching as common. Interestingly, it was also typical for the ELDVs to involve an Attempt to do something, including working toward a specific goal. A participant shared, "I was going to my friend's cottage up a mountain, was snowing hard, had to walk back but never got anywhere."

The remaining subcategories were represented with variant frequency and included (1) Acquiring/ Consuming Behaviors, such as shopping, eating and drinking, getting a gift, and obtaining something;

Table 6. Activities Categories and Subcategories with Case Representation and Frequency

\begin{tabular}{lc}
\hline Category & Frequency \\
\hline 1. Traveling and movement & Typical \\
2. Talking/verbal communication & Typical \\
3. Observing and watching & Typical \\
4. Sport, play, and recreation & Variant \\
5. Harm and injury & Variant \\
6. Domicile/household chores & Variant \\
7. Anticipatory behaviors & Variant \\
8. Attempts & Typical \\
9. Acquiring/consuming & Variant \\
10. Work/school related & Variant \\
11. Destruction/rebuilding & Variant \\
12. Prosocial activities & Variant \\
13. Searching and pursuit & Variant \\
14. Positions of inactivity & Variant \\
\hline
\end{tabular}

This table reports the categories and subcategories from the Activities domain as well as representativeness in the dataset. The frequency labels are determined by CQR (Hill) ${ }^{30}$ and are as follows: General, the category is represented in all or all but one case; typical, occurs between half and less than all; and Variant, equals less than half but more than three cases. 
(2) Anticipatory Behaviors, including planning or preparing for something; (3) Search and Pursuit, looking for someone/something, being followed/chased; (4) Harm and Injury, which was associated with bodily harm, fighting, physical attack, violence to the body, shooting, or killing; and (5) Position of Inactivity, described as sitting still, lying down, sleeping, being stationary, and/or lack of activity.

Setting/location. The final domain includes the setting and backdrop of ELDVs. Individuals typically reported that the environment was Familiar/Known and involved the Natural Environment. Some examples included, "swimming and playing with a dolphin in the ocean," and "I was in a creek...searching for a certain kind of rusty rocks." Similarly, participants typically described settings related to Transportation and Travel (e.g., train stations, buses, planes, etc.).

Typically, people reported ELDVs set in their Home/ Residence, and this included an overall home, structure, or building as well as objects or rooms associated with home, for example, kitchen. In addition, it was typical for participants to describe settings or locations with cues related to Spatial Awareness and Directionality, or where exactly the individual, other characters, and/or objects were within the setting, including references to top, bottom, up, down, side to side, inside/ indoors, and outside/outdoors.

Finally, it was typical for individuals to describe settings related to Institutions of Daily Life. This included Social Gatherings such as weddings, picnics, and graduations, as well as subcategories such as Places of Work, Places of Business, Places of Education, Places of Play (e.g., pool, theatre, cottage, camp, etc.), and Places of Worship. For example, "I was sitting in a kiddie pool outside in the grass with my home health aide." The remaining categories within this domain occurred with variant frequency (Table 7).

\section{Discussion}

This study explored the unique experiences of ELDVs as reported by people receiving hospice homecare. Although some of the findings are congruent with previous research (e.g., comforting ELDVs and activities connecting with family/loved-ones), ${ }^{1,2}$ the results of this analysis showcase a much more expansive and diverse range of interactions, emotions, and activities. This may be due to the use of open-ended questions regarding dream content rather than checklists as in previous studies. ${ }^{2,10}$ Findings align with the conclu-
Table 7. Setting and Location Categories and Subcategories with Case Representation and Frequency

\begin{tabular}{lc}
\hline Category/subcategory & Frequency \\
\hline 1. Transportation and travels & Typical \\
2. Natural environment & Typical \\
3. Familiarity of location & Typical \\
Known/familiar & Typical \\
Unknown/unfamiliar & Variant \\
4. Spatial awareness and directionality & Typical \\
5. Institutions of daily life & Typical \\
Social gatherings & Variant \\
Places of work & Variant \\
Places of business & Variant \\
Places of education & Variant \\
Places of play & Variant \\
Places of worship & Variant \\
Places of education & Variant \\
6. Residence/home & Typical \\
Kitchen & Variant \\
Bedroom/bed & Variant \\
House/home & Variant \\
MISC house stuff & Variant \\
7. Settings associated with death and/or illness & Variant \\
\hline
\end{tabular}

This table reports the categories and subcategories from the Setting and Location domain as well as representativeness in the dataset. The frequency labels are determined by CQR (Hill) ${ }^{30}$ and are as follows: General, the category is represented in all or all but one case; typical, occurs between half and less than all; and variant, equals less than half but more than three cases.

sions of Fenwick and colleagues, who found these experiences "to be far broader than the archetypal image of 'take-away' apparitions or visions at the end of the bed." 9 This is also supported by other research that suggests that dreams may mirror waking life experiences and concerns of healthy and chronically ill and dying people. . $^{8,17,23,25-27}$

The majority of previous ELDV research conceptualizations have focused on deceased loved ones. In this study, participants reported deceased loved ones with equal representativeness as Young people/Babies. Only one other study reported participants experiencing children in ELDVs. ${ }^{11}$ Surprisingly, participants were also equally likely to report an ELDV that included Family, Unknown/Unfamiliar People, and Miscellaneous Characters. These findings broaden previous conceptualizations and suggest that people being featured in ELDVs may be more varied and complex, thus supporting the need to delve further into these experiences.

Participants described interactions within ELDVs that demonstrated Close Connections and Neutral Connections with equal frequency, where previous literature has suggested that the majority of interactions are characterized as close and/or comforting. ${ }^{1,2,9,11}$ It 
is noteworthy that participants also reported conflictual interactions. This significantly expands the understanding of interpersonal dynamics in ELDVs to bear a closer resemblance to waking life. Likewise, the wide range of emotions reported indicates that ELDVs are more varied than previously believed.

Past research documented both the clarity and detail of ELDV experiences. ${ }^{1,11-13}$ The rich descriptions of activities and settings in ELDVs reported here go beyond previous accounts with content that is diverse and broad, and that is reflective of individual perceptions of disease progression, existential distress, and/or life review. As suggested in an earlier study, ELDVs can be an agent for positive psychological growth, ${ }^{32}$ and to assume a distressing/negative ELDV to be less meaningful or inconsequential is an oversimplification.

Understanding the phenomenological experiences of people at the end of life (EOL) is essential to the comprehensive treatment inherent in palliative and hospice care. When appropriate, clinical and interdisciplinary staff can explore ELDVs with patients and their families to gain understanding and build connections. Such experiences may not be universally easy to discuss, yet they present an opportunity to address important aspects of the person's life and dying experience.

Clinical significance has been noted by others who recognized the stigma felt by both the individual and clinicians around the topic of ELDVs. ${ }^{33}$ The importance of addressing ELDVs within a clinical framework is indicated given the prevalence ${ }^{1,10,16,18}$ and potential to provide comfort and closure. ${ }^{1,3,6,15,32}$ It is imperative for clinical staff to build efficacy on how to engage in discussion and validate ELDVs to reduce the stigma around this common EOL experience.

\section{Limitations}

This study primarily included elderly, white, Christian females. The ELDV content of this group may not be generalizable to all and future research should explore ELDVs from other cultural, demographic, and environmental contexts. Second, only some participants recorded their ELDVs between weekly interviews and therefore descriptions may be subject to recall bias. Third, there was no relationship identified between the timing of interviews in relation to patient death and the content of the ELDVs contrary to previous research. ${ }^{1}$ This discrepancy may be related to the setting where data were collected (homecare vs. inpatient) or differences in interview protocols and frequency of in- terviews. Lastly, this study did not specifically collect any data with regards to history of trauma or mental illness. Future studies should more closely focus on the relationship between trauma, mental illness, and existential suffering and how it plays a role in ELDVs.

\section{Conclusion}

The ELDVs are complex subjective experiences with the potential to provide comfort to the dying and insight to clinicians and families. They are valid, meaningful, and encompass a broader range of content and affect than what has been previously reported. Prior research has suggested that peace/comfort can be attributed to "seeing" deceased loved ones and feeling a warm presence. An alternative explanation may be that comfort is a product of making sense/meaning out of the ELDV and/or the ability to safely share the experience with others. Most importantly, it is critical to foster a sense of openness, awareness, and engagement around ELDVs when interacting with those nearing EOL.

\section{Acknowledgments}

The authors would like to thank the people who took the time to participate in this research; even though the time and energy of these people were limited, their words and experiences live on for the authors. The authors would also like to thank their "Dream Team" clinicians who referred participants and/or collected data for this study (Cindy Crossley, Tom Martin, Don Blesnuk, Annie Allen, Ken Keenan, Derek Davis, Bethany Calkins, Kevin McKenzie, Adara Saladino, Christine Ando, Stephanie Kish) as well as their research interns who helped make recruitment calls, collect data, and/or data entry/organization (Amy Watson, Jeff Aldinger, Christopher Moon, Tahleen Lattimer, Ryan Miller). The authors are grateful to Jennifer Breier for feedback to the core data team during the initial stage of analysis. Finally, they would like to thank the Hospice Foundation and its donors for their ongoing support.

\section{Funding Information}

No funding was received for this article.

\section{Author Disclosure Statement}

No competing financial interests exist.

\section{Supplementary Material}

Supplementary Data 


\section{References}

1. Kerr CW, Donnelly JP, Wright ST, et al.: End-of-life dreams and visions: A longitudinal study of hospice patients' experiences. J Palliat Med 2014; 17:296-303.

2. Nosek CL, Kerr CW, Woodworth J, et al.: End-of-life dreams and visions: A qualitative perspective from hospice patients. Am J Hosp Palliat Med 2015;32:269-274.

3. Mazzarino-Willett A: Deathbed phenomena: Its role in peaceful death and terminal restlessness. Am J Hosp Palliat Med 2009;27:127-133.

4. Dos Santos CS, Paiva BSR, Lucchetti ALG, et al.: End-of-life experiences and deathbed phenomena as reported by Brazilian healthcare professionals in different healthcare settings. Palliat Support Care 2017; 15:425-433.

5. Muthumana SP, Kumari M, Kellehear A, et al.: Deathbed visions from India: A study of family observations in Northern Kerala. OMEGA J Death Dying 2011;62:97-109.

6. Levy K, Grant PC, Kerr CW: End-of-life dreams and visions in pediatric patients: A case study. J Palliat Med 2020. DOI:10.1089/jpm.2019.0547

7. Wright ST, Grant PC, Depner RM, et al.: Meaning-centered dream work with hospice patients: A pilot study. Palliat Support Care 2015;13: 1193-1211.

8. Grant P, Wright S, Depner R, Luczkiewicz D: The significance of end-of-life dreams and visions. Nurs Times 2014;110:22-24.

9. Brayne S, Farnham C, Fenwick P: Deathbed phenomena and their effect on a palliative care team: A pilot study. Am J Hosp Palliat Med 2006;23: 17-24.

10. Dam A: Significance of end-of-life dreams and visions experienced by the terminally ill in rural and Urban India. Indian J Palliat Care 2016;22: 130-134.

11. Fenwick $P$, Brayne $S$ : End-of-life experiences: Reaching out for compassion, communication, and connection-meaning of deathbed visions and coincidences. Am J Hosp Palliat Med 2011;28:7-15.

12. Fenwick P, Brayne S: End-of-life experiences: Reaching out for compassion, communication, and connection-meaning of deathbed visions and coincidences. Am J Hosp Palliat Med 2010;28:7-15.

13. Fenwick $P$, Lovelace $H$, Brayne $S$ : Comfort for the dying: Five year retrospective and one year prospective studies of end of life experiences. Arch Gerontol Geriatr 2010;51:173-179.

14. Kellehear A, Pogonet V, Mindruta-Stratan R, Gorelco V: Deathbed visions from the republic of Moldova: A content analysis of family observations. OMEGA J Death Dying 2012;64:303-317.

15. Lawrence $M$, Repede $E$ : The incidence of deathbed communications and their impact on the dying process. Am J Hosp Palliat Med 2012;30: 632-639.

16. Morita T, Naito AS, Aoyama M, et al.: Nationwide Japanese survey about deathbed visions: "My deceased mother took me to heaven." J Pain Symptom Manage 2016;52:646.e5-654.e5.

17. Prince PN, Hoffmann RF: Dreams of the dying patient: An exploration of content. OMEGA J Death Dying 1991;23:1-11.

18. Grant PC, Depner RM, Levy K, et al.: Family caregiver perspectives on end-of-life dreams and visions during bereavement: $A$ mixed methods approach. J Palliat Med 2019;23:48-53.

19. Breitbart W, Alici Y: Agitation and delirium at the end of life: We couldn't manage him. JAMA 2008;300:2898-2910.

20. De J, Wand APF: Delirium screening: A systematic review of delirium screening tools in hospitalized patients. Gerontologist 2015;55:10791099.

21. Inouye SK: Delirium-A framework to improve acute care for older persons. J Am Geriatr Soc 2018;66:446-451.

22. Diagnostic and Statistical Manual of Mental Disorders. Washington, DC: American Psychiatric Association, 2013.

23. Goelitz A: Nurturing life with dreams: Therapeutic dream work with cancer patients. Clin Soc Work J 2001;29:375-385.

24. Shinar YR, Marks AD: Distressing visions at the end of life: Case report and review of the literature. J Pastoral Care Counsel 2015;69: $251-253$.

25. Domhoff GW: The invasion of the concept snatchers: The origins, distortions, and future of the continuity hypothesis. Dreaming 2017;27: 14-39.

26. Schredl M: The continuity between waking and dreaming: Empirical research and clinical implications. In: Dream Research: Contributions to
Clinical Practice. New York, NY: Routledge/Taylor \& Francis Group, 2015, pp. 27-37.

27. Goelitz A: Exploring dream work at end of life. Dreaming 2007. DOI: 10.1037/1053-0797.17.3.159

28. Anderson F, Downing GM, Hill J, et al.: Palliative performance scale (PPS): A new tool. J Palliat Care 1996;12:5-11.

29. Flanagan NM, Spencer G: Informal caregivers and detection of delirium in postacute care: A correlational study of the confusion assessment method (CAM), confusion assessment method-family assessment method (CAM-FAM) and DSM-IV criteria. Int J Older People Nurs 2016;11: 176-183.

30. Hill CE: Introduction to consensual qualitative research. In: Consensual Qualitative Research: A Practical Resource for Investigating Social Science Phenomena. Washington, DC: American Psychological Association, 2012.

31. Hill CE, Knox $S$, Thompson BJ, et al.: Consensual qualitative research: An update. J Couns Psychol 2005;52:196-205.

32. Levy K, Grant PC, Depner RM, et al.: End-of-life dreams and visions and posttraumatic growth: A comparison study. J Palliat Med 2019 (in press).

33. Barbato M, Blunden C, Reid K, et al.: Parapsychological phenomena near the time of death. J Palliat Care 1999;15:30-37.

Cite this article as: Depner RM, Grant PC, Byrwa DJ, LaFever SM, Kerr CW, Tenzek KE, LaValley S, Luczkiewicz DL, Wright ST, Levy K (2020) Expanding the understanding of content of end-of-life dreams and visions: A consensual qualitative research analysis, Palliative Medicine Reports 1:1, 103-110, DOI: 10.1089/pmr.2020.0037.

$\begin{aligned} & \text { Abbreviations Used } \\ & \mathrm{CAM}=\text { confusion assessment method } \\ & \mathrm{CQR}=\text { consensual qualitative research } \\ & \mathrm{ELDV}=\text { end-of-life dream and vision } \\ & \mathrm{EMRs}=\text { electronic medical records } \\ & \mathrm{EOL}=\text { end of life } \\ & \mathrm{PPS}=\text { palliative performance scale }\end{aligned}$

